\title{
Effect of the Volute on Performance of a Centrifugal-Pump Impeller
}

\author{
By R. D. BOWERMAN ${ }^{1}$ AND A. J. ACOSTA2
}

An experimental study of volute influence on radial flow-impeller performance was conducted by operating a single impeller with three different sets of volute vanes. In each case, over-all performance was measured and an internal-flow study within the volute was made. The results show that at their respective design flow rates the influence of the volutes is least and the deviation of performance from the free-impeller operation is small. At off-design flow rates there are major changes in the impeller performance resulting from the presence of the volutes. Large real fluid effects, coupled with a nonuniform velocity pattern at the impeller exit, result in a flow through the volute that does not resemble a potential flow. Even so, the fluid losses through the volute are comparatively small.

\section{Nomenclature}

The following nomenclature is used in the paper:

$A_{3}=$ outlet area of impeller

$b=$ impeller-passage height

$c=$ absolute fluid velocity

$c_{m}=$ absolute meridional component of fluid velocity

$c_{u}=$ absolute tangential component of fluid velocity

$C=$ static-head coefficient $=H_{s} g / u_{2}{ }^{2}$

$g=$ gravitational constant

$H_{T}=$ total head (referenced to total head at impeller inlet)

$H_{S}=$ static head (referenced to total head at impeller inlet)

$Q=$ flow rate

$r=$ radius from impeller axis

$r_{v}=$ radial co-ordinate of volute vane

$\mathrm{R}_{\mathrm{c}}=$ Reynolds number $=r_{2}{ }^{2} \omega / \nu$

$T=$ torque

$u=$ impeller tangential velocity $=\omega r$

$\beta=$ impeller-vane angle

$\beta_{v}=$ volute-vane angle

$\eta=$ efficiency $=\frac{\phi \psi}{\tau}$

$\theta=$ angular co-ordinate of volute vane; absolute angular coordinate

$\rho=$ fluid density

$\tau=$ torque coefficient $=\frac{T}{\rho A_{2} u_{2}{ }^{2} r_{2}}$

$\phi=$ flow-rate coefficient $=c_{m 2} / u_{2}=Q / A_{2} u_{2}$

$\phi_{c}=$ impeller-design flow-rate coefficient

$\psi=$ total-head coefficient $=H_{T} g / u_{2}{ }^{2}$

1 Research Engineer, U. S. Industries Research \& Development Corporation, Los Angeles, Calif. Assoc. Mem. ASME.

2 Assistant Professor of Mechanical Engineering, California Institute of Technology, Pasadena, Calif. Assoc. Mem. ASME.

Contributed by the Hydraulic Division and presented at a joint session of the Hydraulic and Gas Turbine Power Divisions at the Semi-Annual Meeting, Cleveland, Ohio, June 17-21, 1956, of The American Society of Mechanical Engineers.

Note: Statements and opinions advanced in papers are to be understood as individual expressions of their authors and not those of the Society. Manuscript received at ASME Headquarters, March 16 , 1956. Paper No. 56-SA-45.

\author{
$\omega=$ angular velocity of impeller \\ $\nu=$ kinematic viscosity \\ Subscripts \\ $1=$ impeller inlet $\left(\beta_{1}\right)$ \\ $2=$ impeller outlet $\left(\beta_{2}, r_{2}, u_{2}, A_{2}, c_{m 2}\right)$ \\ $i=$ impeller only $\left(\psi_{i}, \eta_{i}\right)$ \\ $p=$ "whole pump" (impeller with volute) $\left(\psi_{p}, \eta_{p}\right)$ \\ $x=$ design quantity $\left(\psi_{x}, \phi_{x}, \eta_{x}\right)$ \\ $v=$ refers to volute quantity $\left(\psi_{v}, C_{v}\right)$
}

\section{INTRODUCTION}

The pump-research program of the Hydrodynamics Laboratory of the California Institute of Technology has been directed towards determining basic design information by systematically studying the performances of pump components and by correlating performance with internal-flow phenomena. Previously, extensive work has been conducted on centrifugal impellers operated with a vaneless diffuser; i.e., an annular passage of constant breadth. Thus, polar symmetric conditions existed for the impeller over the complete range of flow rates. An impeller operated in this manner is herein termed a free impeller. In the present work, effects of the volute case on the aggregate pump have been studied by successively combining three simplified two-dimensional volute shapes with a single impeller (previously studied as a free impeller) and conducting over-all performance and internalflow experiments on the combinations.

The information sought can be divided roughly into two categories: $(a)$ the over-all performance for comparison of the different impeller-volute combinations and determination of the volute effect on the impeller itself; and $(b)$ the nature of the flow through the volute, its agreement with potential theory, and its correspondence with the features of the over-all performance. At a given speed of rotation, the over-all performance is described completely by the head and torque as functions of flow rate from which the water power and efficiency can be calculated. The flow in the volute is determined by measurements of the total-head and static-head distributions from which velocity can be calculated.

With regard to the over-all performance of the impeller-volute combinations, a distinction is made between impeller performance based on averaged head measurements made at the impeller exit and pump performance based on averaged head measurements made at the volute exit. Furthermore, as the definition of pump performance indicates, the "pump" of this test is not directly comparable to a commercial pump, as the latter also includes final collection of the discharge into a single pipe with the consequent additional adjustment of the flow pattern to uniform pipe flow. Thus, the complete pump is subject to additional mixing losses which are not accounted for in the volute losses reported here. It should be noted also that, unlike commercial tests, the torque resulting from disk friction and other mechanical losses is not included in the present tests.

\section{Design and Construction}

The impeller employed in the investigation was a two-dimensional design with a 20-deg inlet angle and a 23.5-deg outlet angle, and has been described thoroughly in previous publications $(1,2$, 


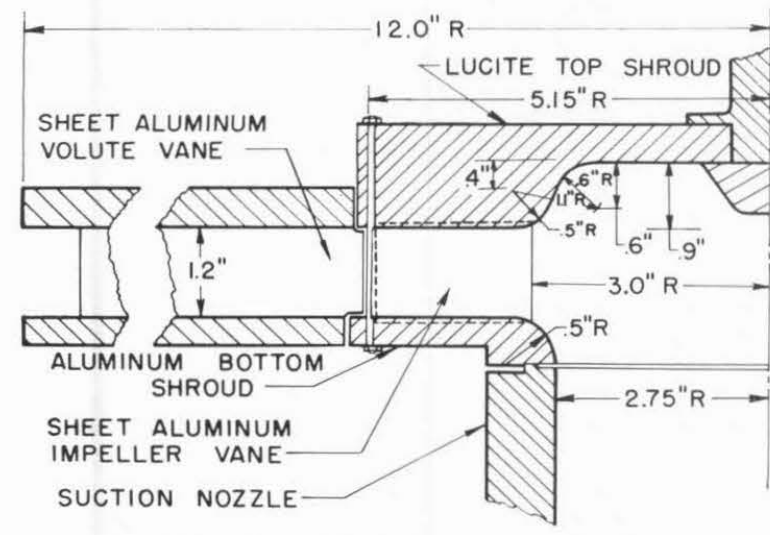

Fig. 1(a) Axinl Cross Section of Test Pump



Fig. 1(b) Layout of Volute Vanes in Diffuser Shrouds. Position of Piezometer Taps Shown as Follows:

Indicates static tap
Indicates static tap and total-head probe

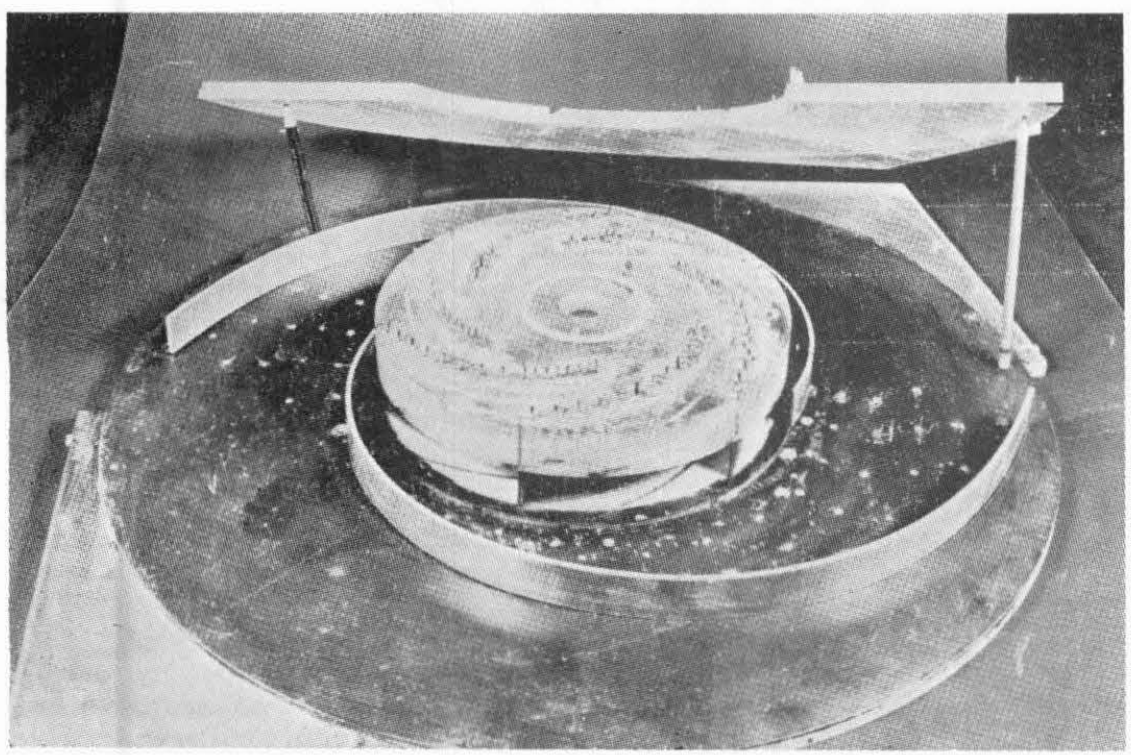

Fig. 2 Assembly View of Pump Components
3). ${ }^{3}$ The two-dimensional volute cases were formed by fitting the volute vanes between parallel diffuser shrouds, as seen in Figs. $1(a),(b)$, and 2. Double-volute construction was chosen to meet space limitations and to make an added study of flow symmetry. It should be noted that the volute vanes extend inward quite close to the impeller vanes. This close proximity is not common in commercial designs; however, for the purposes of this study, the maximum effect of the volutes was desired.

The volute-vane shapes were derived by considering the elementary theory of constant angular momentum. In two dimensions this yields the equation of the familiar logarithmic spiral

$$
r_{v}=r_{2} e^{\frac{\phi x \eta x}{\psi x} \theta}
$$

where $r_{v}$ and $\theta$ are the radial and angular co-ordinates of the volute vane, $\phi$ and $\psi$ are dimensionless coefficients of the flow rate and head, respectively, and $\eta$ is the efficiency. Calculation of a volute shape was made by choosing a volute-design flow rate $\phi_{x}$ and taking the corresponding $\psi_{x}$ and $\eta_{x}$ data from the free-impeller characteristies. The ratio $\phi_{x} \eta_{x} / \psi_{x}$ which is the tangent of the volute-vane angle is also the tangent of the actual flow-discharge angle from the impeller at the chosen flow rate. The three sets of volutes resulted from the choice of $\phi_{x}$ equal to 75 , 100 , and 120 per cent of the free-impeller design point, $\phi_{e}$. All pertinent dimensions and design constants are given in Table 1.

The volute vanes were formed of $1 / 8$-in. aluminum sheet, $1.2 \mathrm{in}$. wide, bent to match metal templates cut to the $r_{v}$ and $\theta$-co-orordinates. The vane leading edges were rounded. The templates also were used to scribe the vane positions on the diffuser shrouds and the vanes were held in position with screws. A single set of shrouds was made to accommodate all three sets of vanes.

\section{EXPERIMENTAL WORK}

Test Program. The following experiments were made on each of the impeller-volute combinations:

I Static-head measurements around the impeller periphery.

II Total-head measurements at the radial probe stations in the volute passage. Total-head measurements were made at five elevations.

III Input-torque measurements.

Additional pressure-distribution measurements on the volute vanes and tuft studies in the volute passages were made but are not reported herein.

Instrumentation. The Laboratory facilities have been described thoroughly in previous publications $(1,2)$ and will not be further recorded here. The static-pressure measurements of Test I were made by means of 42 static piezometer taps $(0.020$ in. in diam) that were spaced around the impeller periphery $3 / 16$ in. from the impeller exit.

The total and static-pressure data needed in Test II were measured by static holes and total-head probes set into brass inserts which were set into radial slots machined in the top shroud. Separate inserts for static and total-head measurements were used. The angular direction and elevation of the total-head probes were maintained by pouring melted wax around the base of the probes. The total-

3 Numbers in parentheses refer to the Bibliography at the end of the Paper 
TABLE 1 TABLE OF CONSTANTS

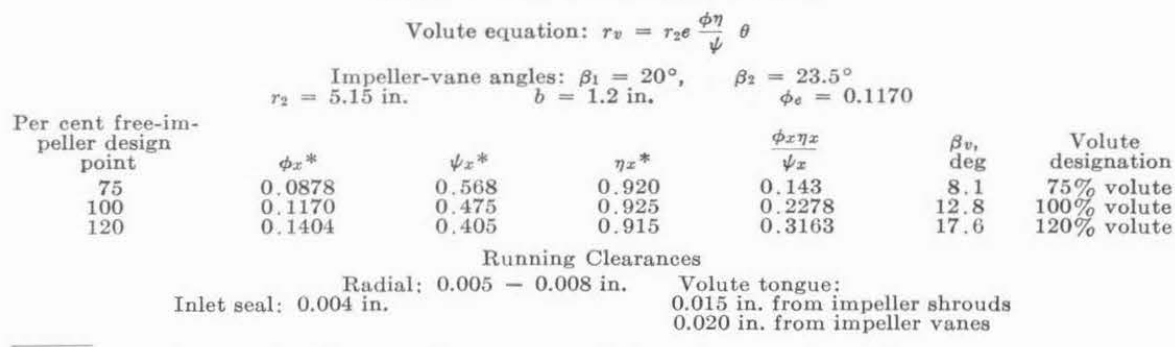

* Measured free-impeller characteristics, $\psi$ measured $1 / 16$ in. from impeller exit.

head-tube proportions were slightly unusual. The design and calibration are given in Fig. 3 . The location of the static-pressure holes and inserts for total and static-pressure determination is shown in Fig. $1(b)$. The completely instrumented volute is shown in Fig. 4, together with the multi-tube manometers used to read the pressure signals.

All pressure measurements were referenced to the total head at the inlet of the impeller.

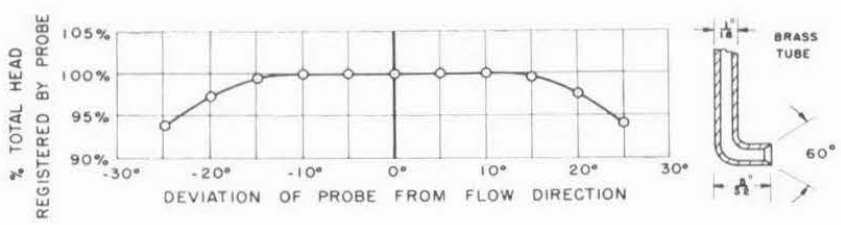

Fig. 3 Angle Sensitivity of Total-Head Probes



Fig. 4 Assembly of Pump Components
Torque measurement was accomplished by balancing the reaction torque of the motor case with weights suspended over a pulley from a lever arm. The balance position was indicated by a Statham displacement gage and fixed-bridge circuit.

Procedure. All tests were conducted at $150 \mathrm{rpm}$ over an established set of flow rates. To minimize the effect of probe interference, all static-head data were taken independently of the totalhead data and total-head data were taken with the probes evenly divided between the two volute passages to maintain flow symmetry.

The torque tests required careful technique as the bridge drift and general laboratory vibration contributed to scatter in the readings. As the torques involved at the low $\mathrm{rpm}$ were small $(0.2-0.5 \mathrm{ft}-\mathrm{lb})$, the percentage error was large. Continual checks were made on the bridge zero and statistical methods were applied to the taking of each reading. As only the torque due to the impeller-vane action was wanted, the tare torque which included the motor windage and the fluid-disk friction on the outside of the impeller had to be measured. This was done by running the impeller empty but with a temporary basin holding water atop the impeller, simulating the operating condition. Duplication of actual running conditions was difficult and the reading was sensitive, but identical technique was employed for all the torque tests. Estimated final torque error is within \pm 2 per cent.

\section{Results and Discussion}

Presentation of Data. All quantities are reported as dimensionless coefficients as defined in the nomenclature. The curves of Figs. $5(a),(b)$, and $(c)$ represent the impeller head as a funetion of the angular position from the volute tongue. Each of these values is the average of five head readings taken over the breadth of the impeller. The over-all impeller head is the average of the data of Fig. 5 and it should be recognized that these averages cannot be accurate where the curves differ widely. The over-all pump head (Fig. 13) was obtained by an integrated average of the head values over the volute exit. Velocity coefficients were calculated from the difference in total head and static head, and it was necessary to assume that the static head at the top shroud was a representative of that across the passage depth.

With regard to free-impeller-head values, some explanation is required as to the manner in which they were obtained. In Fig. 6, curves $A$ and $B$ are the result of earlier work on the impeller where curve $A$ represents measurements made $1 / 16$ in. away from the impeller-vane exit and curve $B, 3 / 8$ in. away. The difference of these curves was considered in reference (1), wherein at low flow rates it was decided that large mixing losses occur immediately following the impeller exit. Values from curve $A$ were used in calculating the volute shapes. The data for curve $C$ were currently taken in an identical manner as the data with volutes; i.e., ${ }^{5} / 32$ in. away from the impeller exit. Curve $C$, generally in accord with curves $A$ and $B$, is taken as the free-impeller performance for comparison with the data of the volutes. 


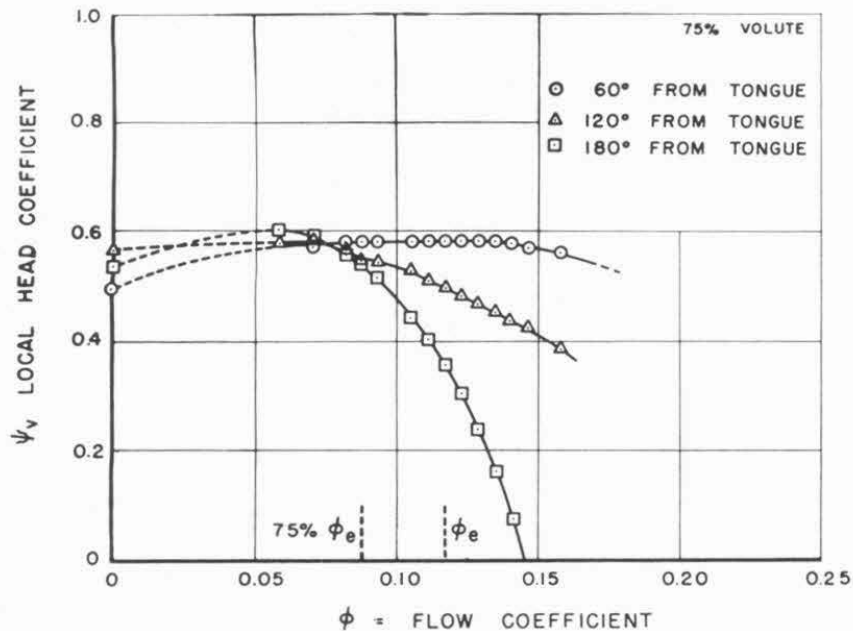

Fig. 5(a)



Figs. 5 $(a, b, c)$ Impeller-Head Coefficient Measured at 60, 120 , 180-Deg Positions From Volute Tongue for Three Volute

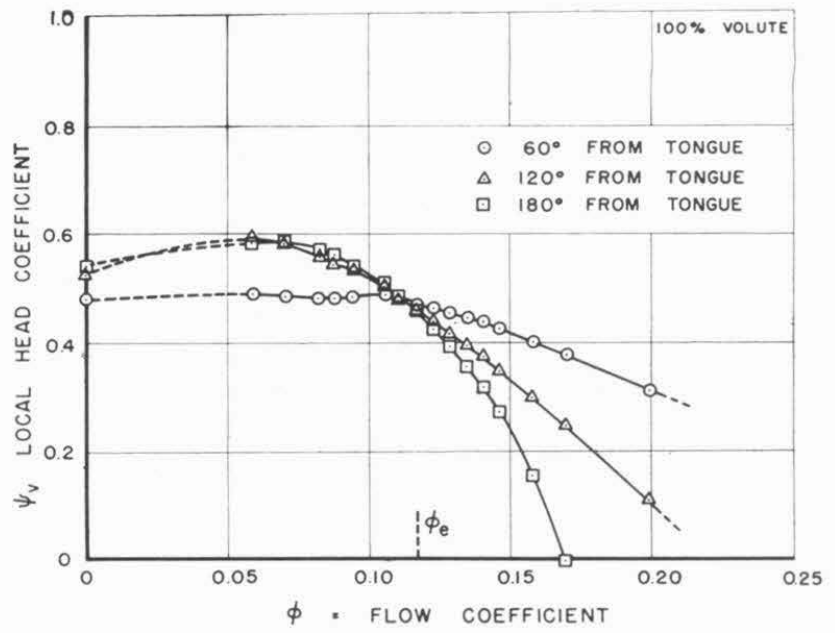

Fig. 5(b)



Fig. 6 Free-Impeller Developed-Head Coefficient Measured at Three Distances From Impeller Exit

\section{Effect of Volutes on Performance}

Over-All Results. The experimental results show that the volute shape is quite prominent in determining the performance characteristics of a pump. The significant result is that the performance is controlled by the influence of the volute shape on the impeller operation rather than by the addition of losses to the fluid within the volute. Conclusive evidence that the impeller operates differently with different volute shapes is found in the torque curves (Fig. 7). Although there is not too much difference in the torque curves at low flow rates, above $\phi=$ 0.09 the curves are distinct, and at $\phi$ above 0.14 there is considerable variance. The 75 and 100 per cent combinations generally require somewhat greater torque than the free impeller, whereas the 120 per cent combination torque requirement is about the same as the free impeller.



Fig. 7 Torque Coefficient and Pump Water Power Coefficient for Three Volute Shapes 


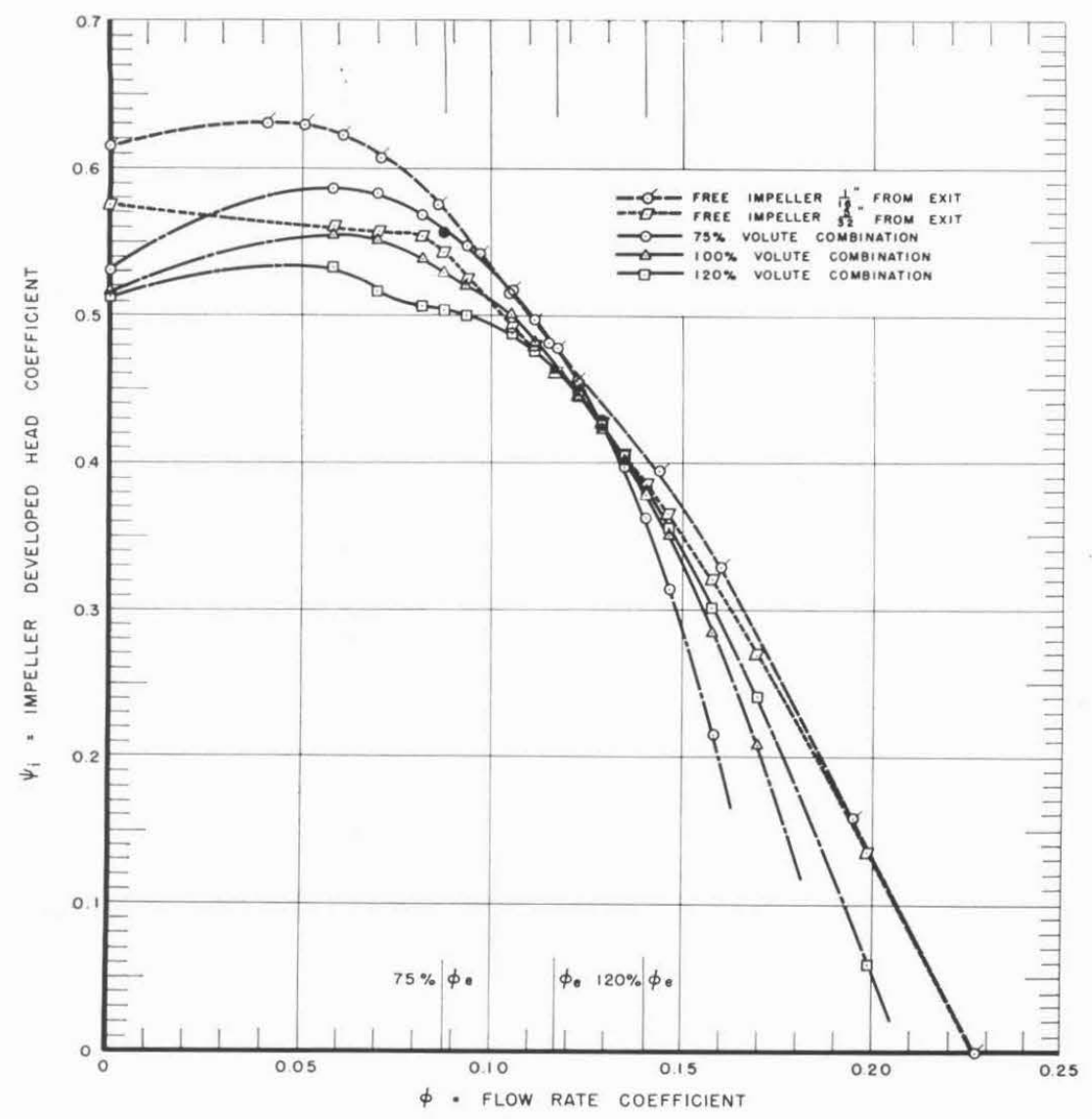

Fig. 8 Impeller Developed-Head Coefficient for Three Volute Shapes and Free IMPELLER

Fig. 9 Impeller Efficiency for Three Volute Shapes aNd Free Impeller

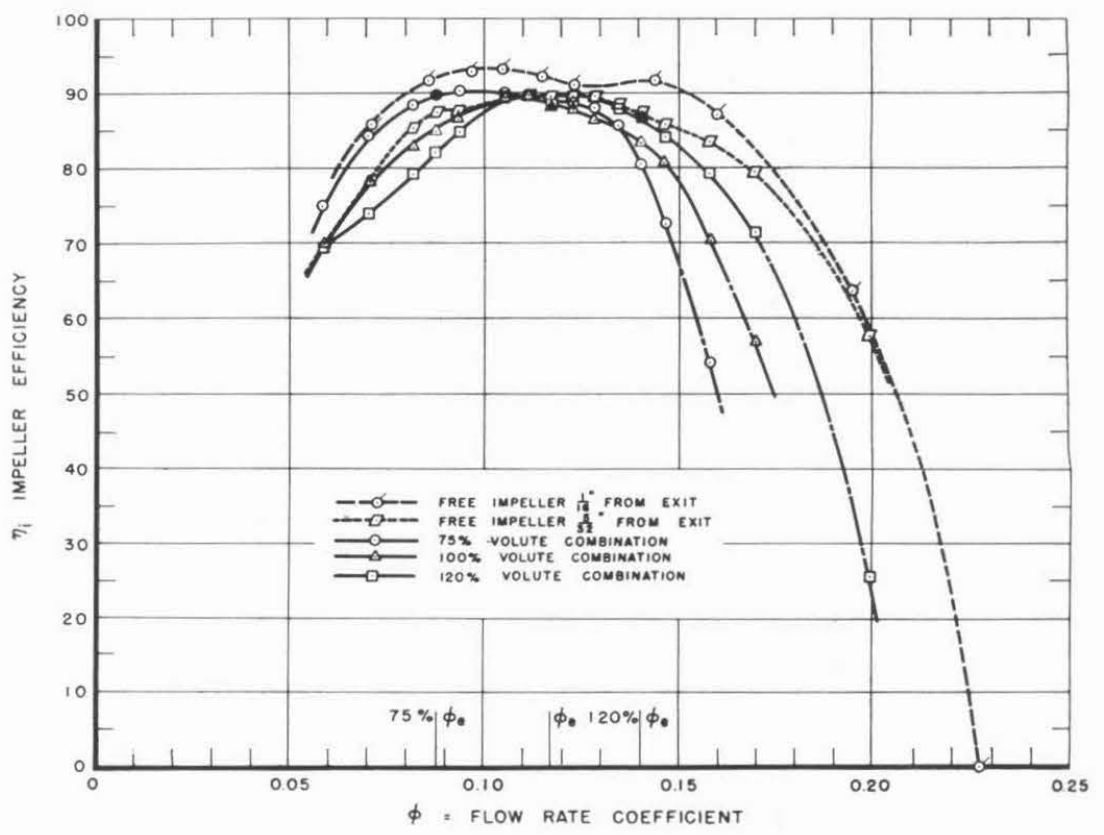



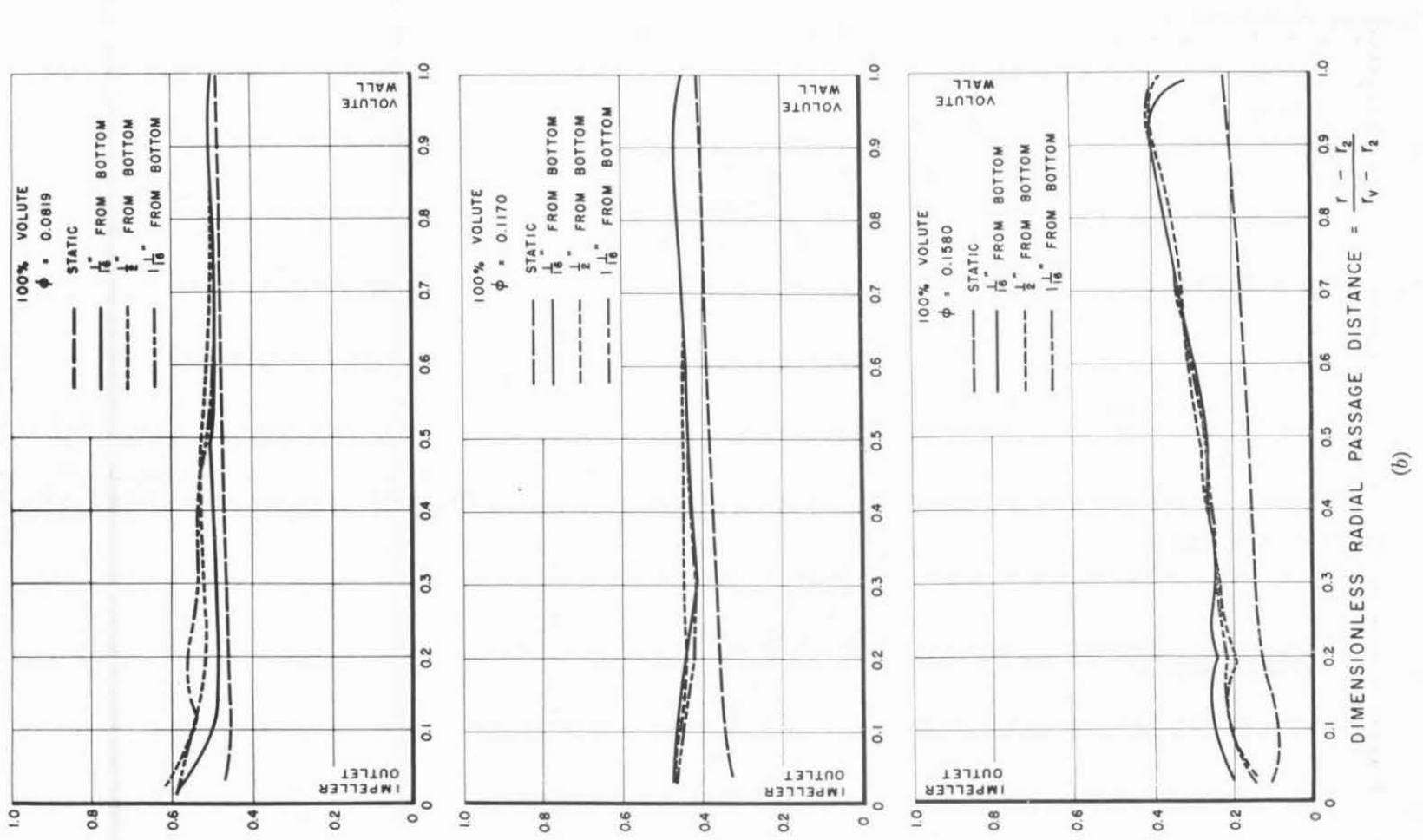

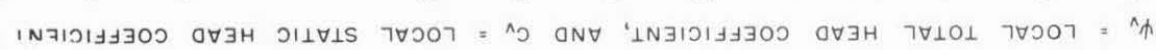



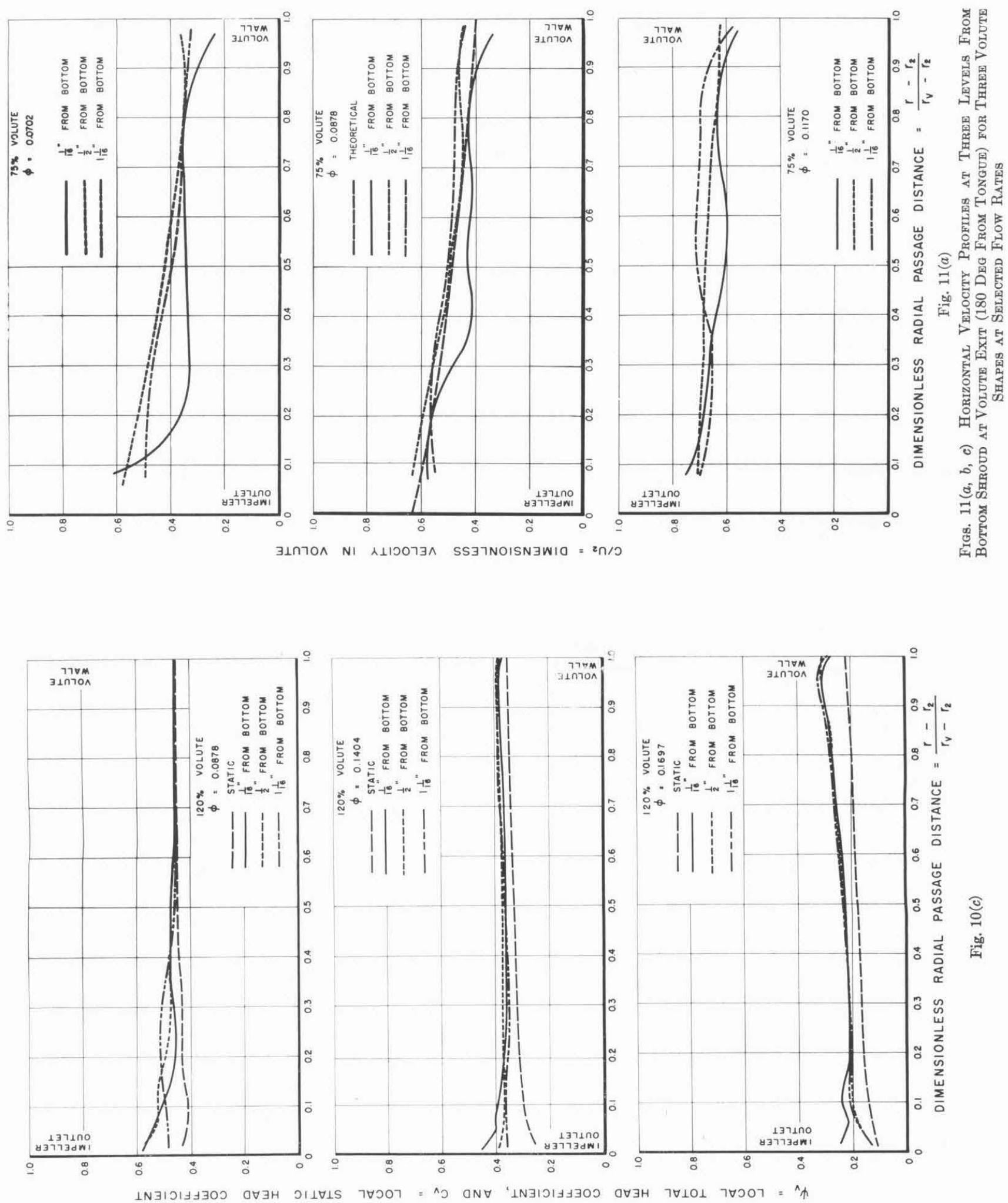

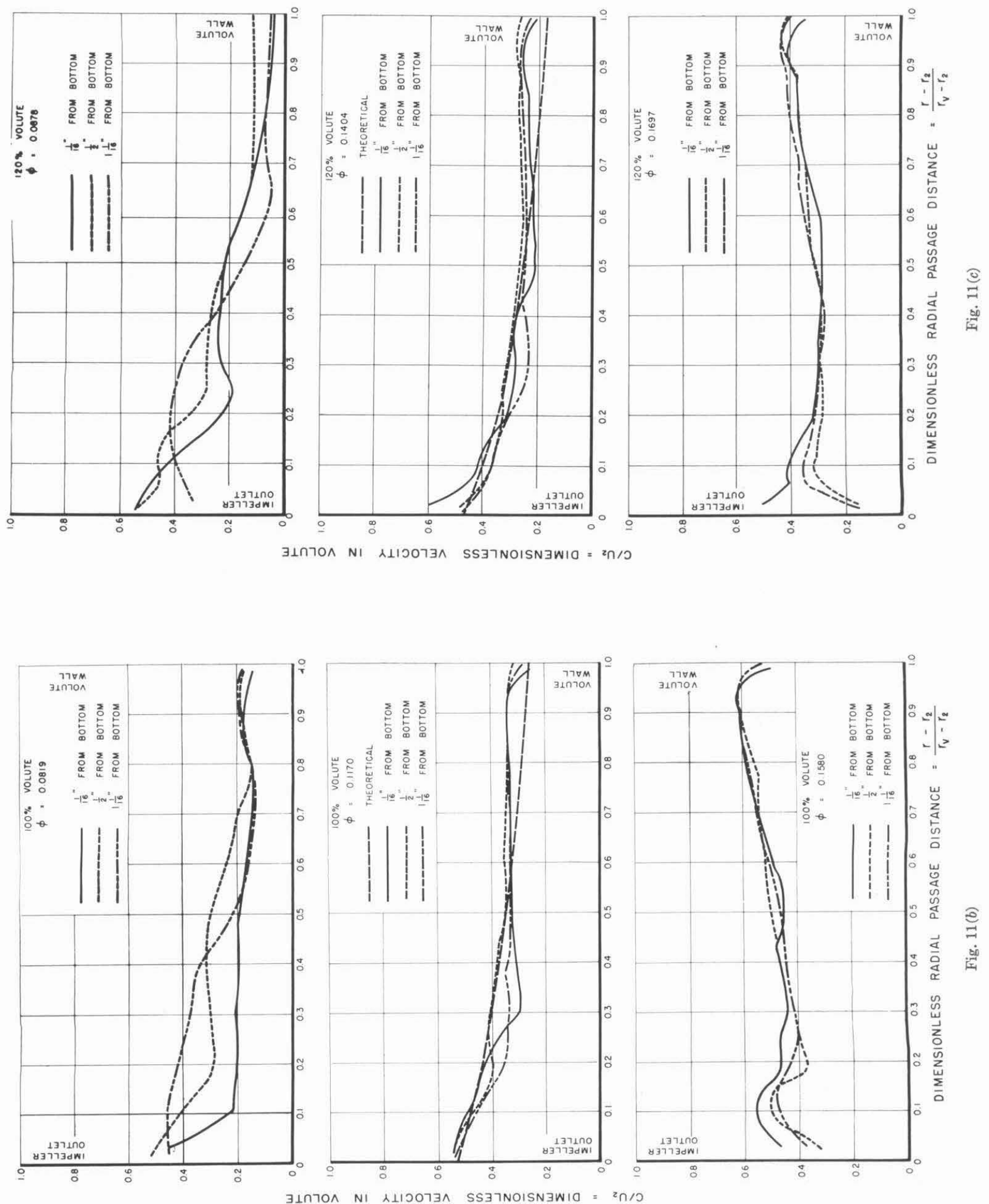

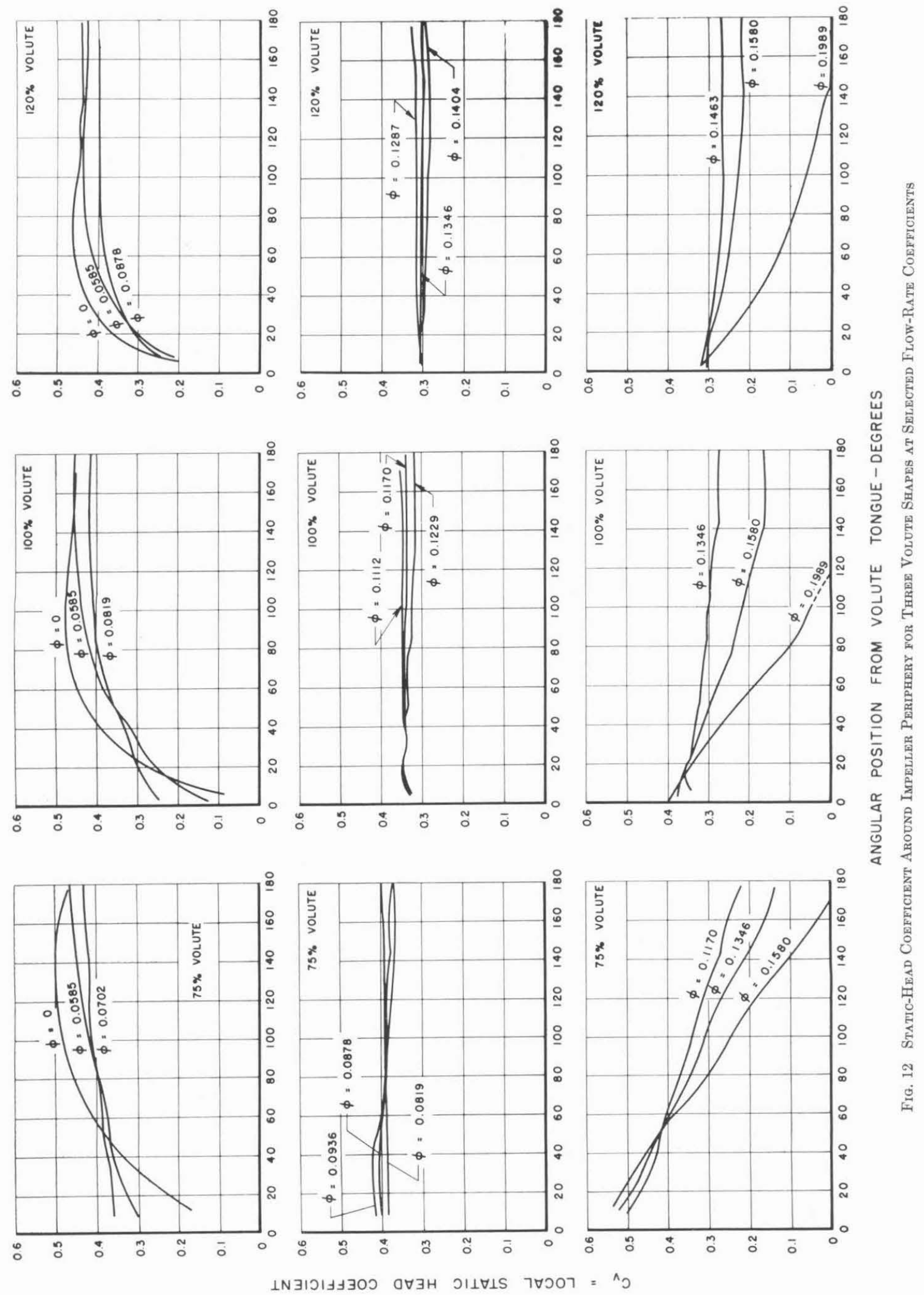
Additional evidence of volute effect on impeller performance is given by the impellerhead curves (Fig. 8). The heads are similar over the span of the design points; however, at low flow rates and high flow rates, there are large differences in the head curves. In general, it can be concluded that each volute causes different loss distribution within the impeller and immediately following it for these reasons: At very low flow rates each of the four cases must have a different impeller loss because the heads are quite different even though there is little change in the torque. At high flow rates, the impeller heads of the 120,100 , and 75 , per cent combinations successively decrease and are lower than the free-impeller values; however, the torques progressively increase, all being as high or higher than the free-impeller requirement. The impeller-efficiency curves (Fig. 9) reflect the effect of these losses.

Effect of Volutes at Design Points. The operation of each volute-impeller combination is optimum at approximately the individual volute design flow rate. The heads produced by the 100 and 120 per cent combinations are almost the same as the free-impeller head measured $5 / 32$ in. away from the impeller exit. At the flow rate $0.75 \phi_{e}$, the free-impeller head measured at $5 / 32$ in. away is below that measured $1 / 16$ in. away, and the 75 per cent volute combination produced a head between these two free-impeller values. Evidently in the 75 per cent case the presence of the volute alleviates the conditions causing the losses immediately following the impeller.

The internal-flow data also support the fact that the volutes operate best at their design points. In each case, the radial surveys of total and static head and their consequent velocity distributions are smooth, Figs. 10 and 11, and the impeller total heads, measured at the various angular positions, Fig. 5, are nearly equal.

Likewise, the static pressure distribution around the impeller periphery is quite uniform, Fig. 12. These results indicate that the volute can be designed without taking into account the effect of friction, at least for the relatively high Reynolds numbers (2.4 $\times 10^{5}$ ) of these tests.

Complete Pump Performance. The pump-performance curves (Figs. $7,13,14$ ) are seen to be not significantly different from the impeller-performance curves. There are some losses as the fluid passes through the volute, as demonstrated by the totalhead curves compared in Fig. 15. At the volutedesign points, the losses through the volutes in each case are approximately 3 per cent of the total head of the impeller (which amounts to about 20 per cent of the volute exit-velocity head). However, except at the volute-design flow rates, the losses incurred in the volute are of a lesser magnitude than the changes in impeller performance of the three volute combinations. All impeller-volute combinations have nearly

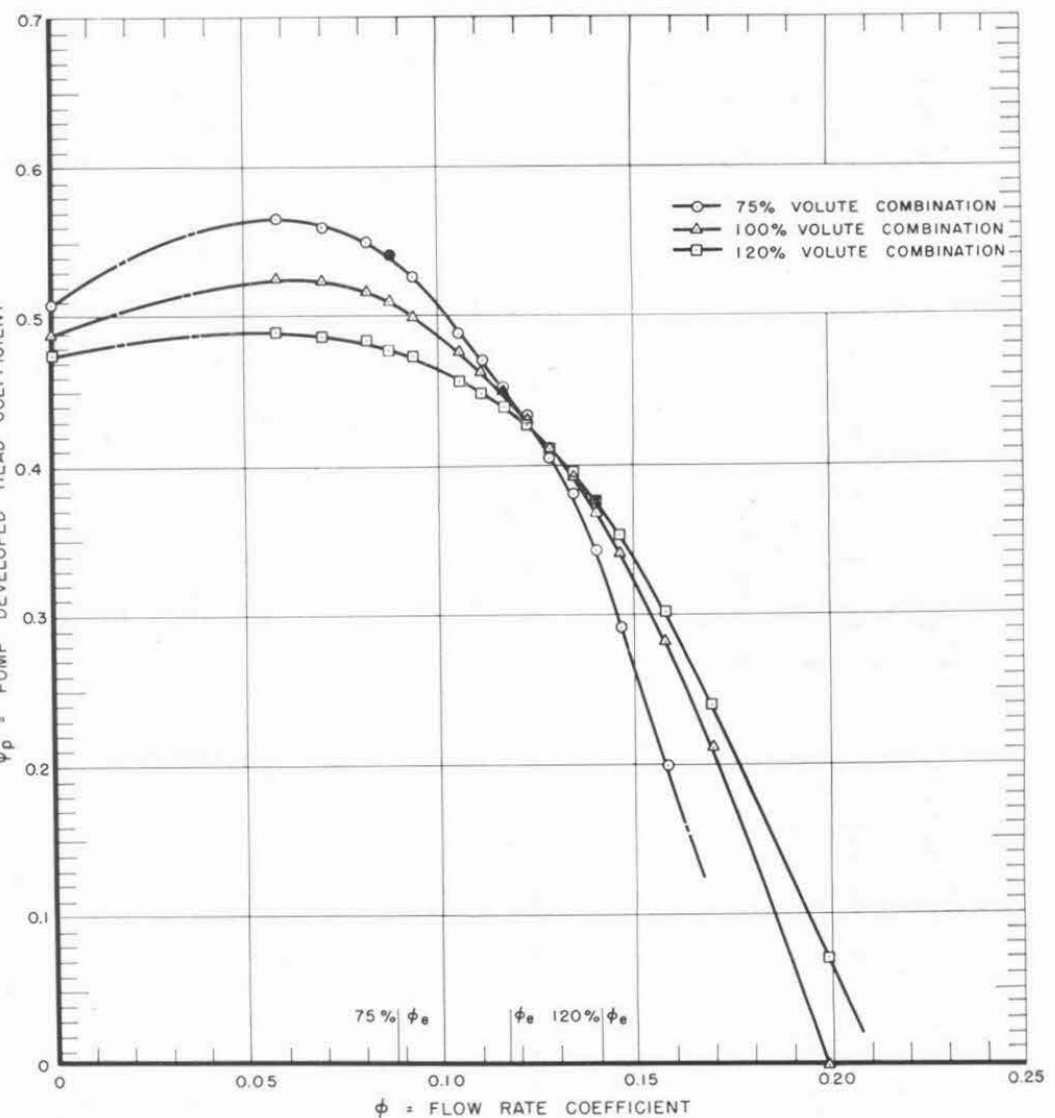

ig. 13 Pump Developed-Head Coefficient for Three Impeller-Volute ComBINATIONS

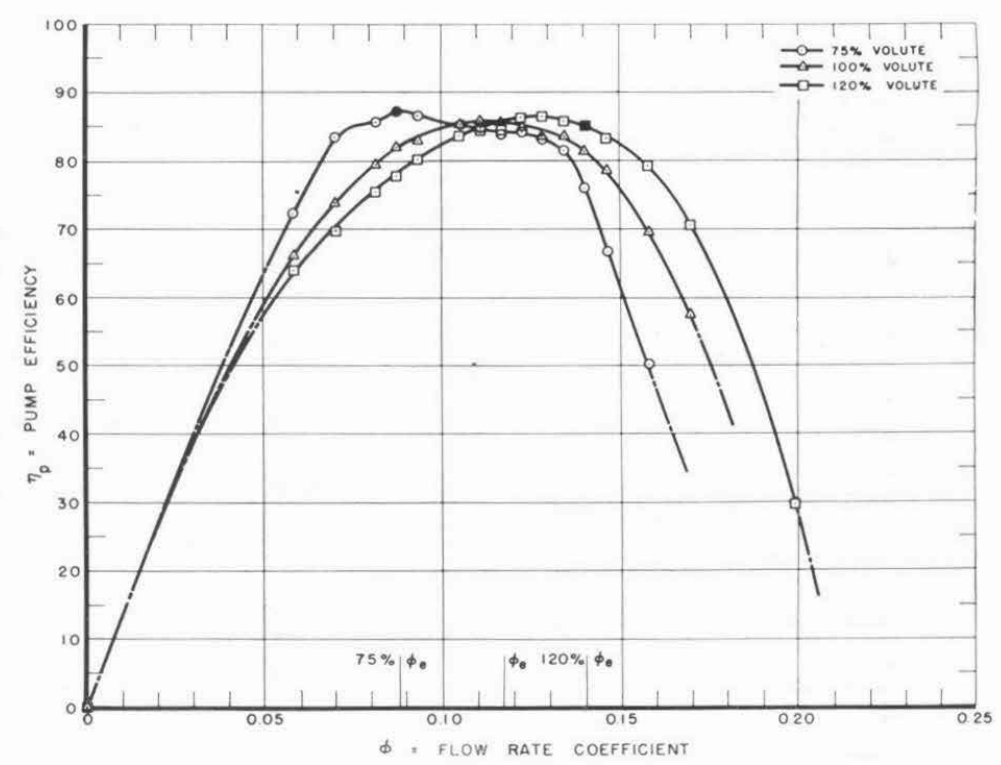

Fig. 14 Pump Efficiency for Three Impeller Volute Combinations 

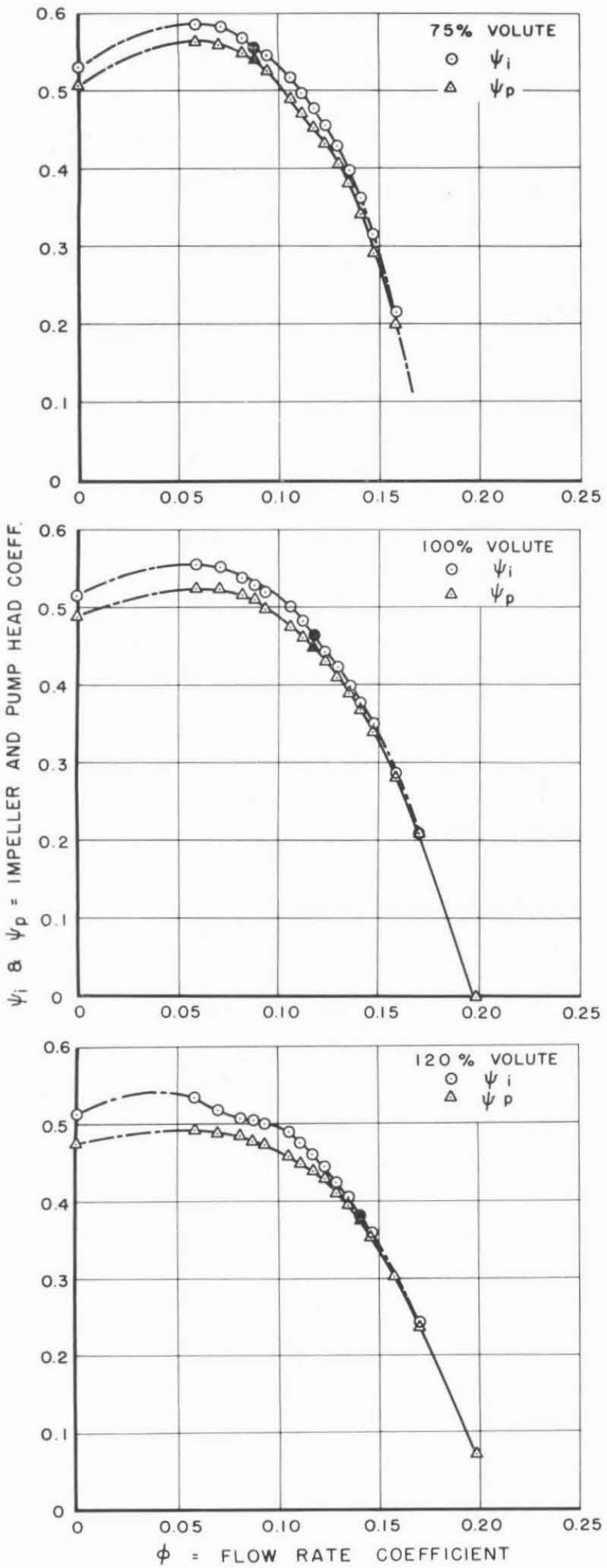

equal best efficiency (about 87 per cent) which is probably due to the fact that the design points of the volutes are within the high-efficiency region of the free impeller. It is not likely that a volute designed below the high-efficiency region of the impeller would give good performance.

Flow Patlerns Through the Volute. As happens in many other flow problems, the flow in the volute has an approximate potential flow static-pressure distribution, but the velocity distribution is not at all that predicted by theory. The local total and statichead curves and horizontal velocity-profile plots, Figs. 10 and 11, show that at design points the velocity diminishes to some degree with increasing radius to about one third of the volute-passage width, and from there on to the volute vane it is nearly constant. Potential flow requires the velocity to diminish inversely as the radius. Furthermore, over the entire range of flow rates there appears to be a region of high-velocity flow along the concave side of the volute vane that would not ordinarily be expected. It is believed that this result is due to a secondary flow of the high-energy fluid coming from the impeller-shroud boundary layer. Considerable flow disturbance is eaused by this discharged-impeller boundary layer, as can be seen in Figs. 10, 11, and 16. The effect is notably more pronounced on the bottom surface as the bottom impeller shroud has a larger diameter. The fluid elements with varying total energy necessarily mix at the impeller exit, and this process accounts for some of the observed losses.

\section{Discussion}

It would be of interest to be able to predict, roughly, the direction and magnitude of the volute-impeller interference effects. Early investigators, e.g., Straszacker (4), have made attempts in this direction by assuming the absolute flow to be irrotational and inviscid, so that potential theory could be used. Straszacker was concerned chiefly with the flow and streamline picture at the impeller exit rather than with performance calculations.

At off-design conditions high velocities can occur at the volute tongue and these disturbances may cause substantial deviations in the velocity pattern. If the impeller is considered to have an infinite number of blades, it can be shown (5) that these deviations will give rise to a nonuniform total head around the periphery of the impeller and, as a consequence, the flow leaving the impeller will not be irrotational. The same conclusions also may be obtained if the impeller is considered to have a finite number of vanes; however, the discussion proceeds more easily with an infinite number of vanes.

The influence of the volute on the impeller performance (from an elementary point of view) comes from the nonuniform distribution of radial velocity around the impeller. If it may be assumed that the infinite number of vanes perfectly guides the relative flow, then the torque required can be determined from the momentum law. From this computation it is easy to show that any variation in radial velocity around the impeller leads to a decrease in required torque. If the impeller efficiency were unchanged, the impeller head would thus be reduced.

The foregoing deduction is not borne out by the present experiments. Practically all cases show an increase in the torque required by the impeller. It must be concluded that either the foregoing simple picture is inadequate or the principal assumption, i.e., that the flow is perfectly guided by the impeller vanes, is erroneous. Probably both situations occur. In potential flow, with a finite number of blades, the Kutta condition is applied at the impeller tips and replaces the perfect guidance of the infinite-vane case. The presence of the volute tongue gives rise to

Fig. 15 Impeller and Pump-Head Coeffictents Compared for Three Volute Shapes; $\psi_{i}$ Denotes Impeller Head; $\psi_{p}$ Denotes PUMp Head 

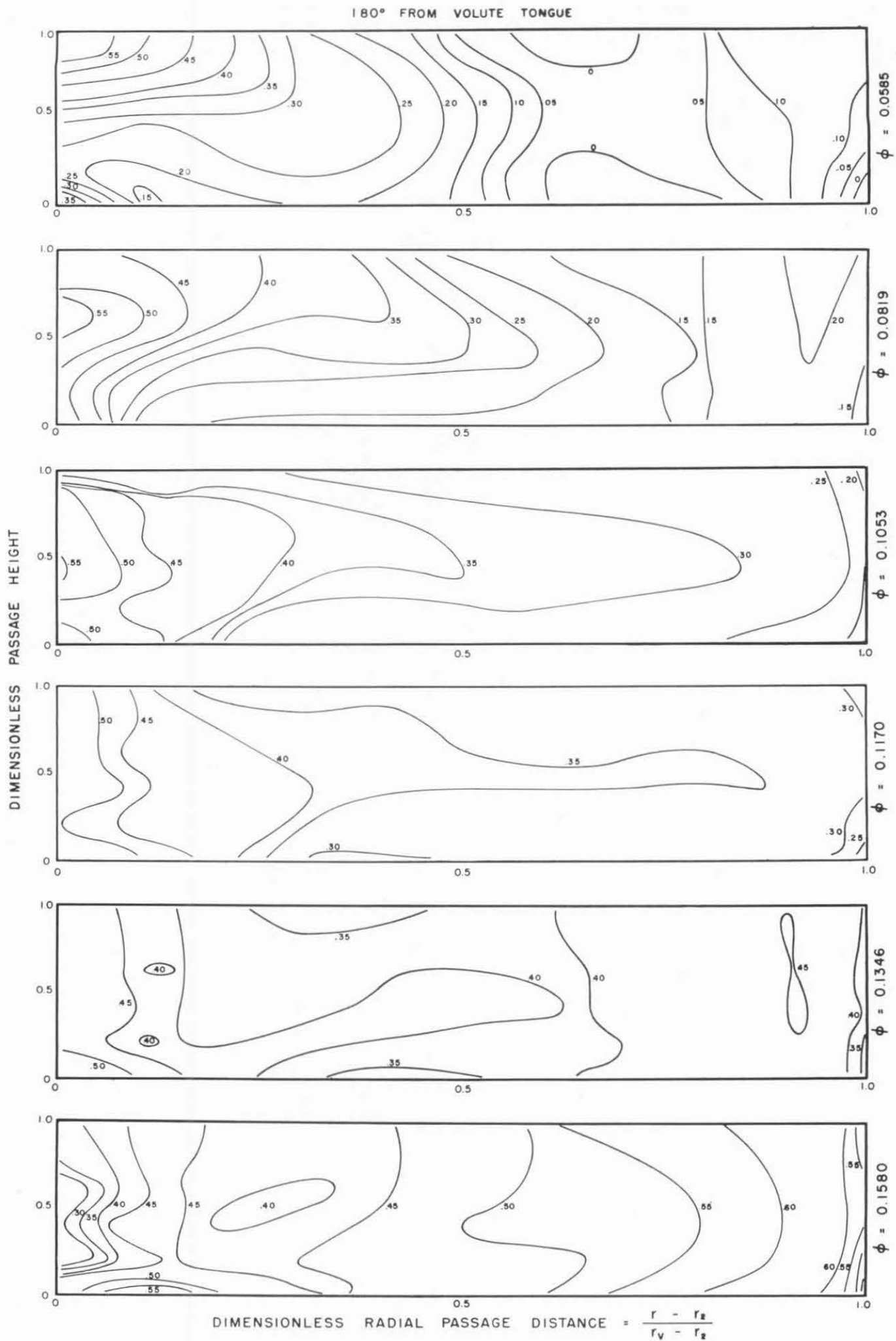

Fig. 16 Velocity Contours $\left(c / u_{2}=\right.$ Const) for 100 Per Cent Volute at 180 Deg From Volute Tongue at Selected Flow Rates 
trailing vorticity and nonsteady flow patterns in both the relative and absolute flow patterns. Their precise influence on torque is not known, although the situation is qualitatively similar to the infinite-vane case. Since the volute vanes were only about $1 / 16$ in. from the impeller, enormous nonsteady disturbances could occur there, and it does not seem unreasonable that the realfluid flow cannot always adjust itself to stream smoothly off thebladetips. If the condition of perfect guidance (for an infinite number of blades) is relaxed, then it is possible for an increase in torque to occur. This result also follows from the intuitive notion that near the volute tongue, the absolute flow leaving the impeller is determined by the volute-vane angle. Thus, at high flow rates, the tangential component of absolute velocity (and thus torque) would be greater than in the free-impeller case. If it were not for a simultaneous decrease in impeller efficiency, a larger average head would result. An inspection of Fig. 5 does show that the head measured $60 \mathrm{deg}$ from the volute tongue is consistently high for the high flow rates and low for the low flow rates, showing that the head there is controlled by the volute vanes.

This discussion is admittedly speculative in part and should be subjected to further investigations. It appears, however, that potential theory cannot be used to describe the flow at off-design conditions. At the present time no satisfactory method of accounting for off-design performance is available.

\section{Conclusions}

From a series of experiments on a two-dimensional impeller and simplified volute shape, the following observations can be made:

1 For efficient operation, the volute must be matched to the impeller at the desired design flow rate.

2 At the design point of the volute, its influence is relatively small and it can be designed without considering the effect of friction at the Reynolds numbers of these experiments.
3 At off-design conditions large changes in pump performance occur that are due primarily to changes in impeller performance.

4 There are large real fluid effects in the volute flow that arise from the discharge of the rotating boundary layer into the volute channel. The resulting velocity distributions are only qualitatively similar to a potential flow; i.e., constant angular momentum at design conditions and depend markedly on flow rate.

In the present experiments, the maximum influence of the volute vanes has been studied. The degree of volute-impeller interference is, of course, dependent upon the spacing between them. It is hoped that investigation of this variable, as well as that of Reynolds number, will be undertaken in the future.

\section{ACKNOWLEDGMENT}

This work was supported by the Office of Naval Research. Reproduction in whole or part is permitted for any purpose of the United States Government.

\section{BIBLIOGRAPHY}

1 "Evaluation of a Two-Dimensional Centrifugal Pump Impeller," by J. H. Beveridge and D. A. Morelli, ASME Paper No. 50A-147, unpublished.

2 "Pressure Distributions on the Vanes of a Radial Flow Impeller," by D. A. Morelli, Transactions of the Heat Transfer and Fluid Mechanies Institute, 1950, p. 73; Stanford University Press, Stanford, Calif., 1950.

3 "An Experimental Study of Centrifugal Pump Impellers," by A. J. Acosta and R. D. Bowerman, California Institute of Technology, Hydrodynamics Laboratory Report No. E-19.8, August, 1955 .

4 "Die Ermittlung der Strömungsverhaltnisse in Spiralgehausen," by R. Straszacker, Ingenieur-Archiv, VI-3-1935, pp. 157-182.

5 "Effect of the Volute on Performance of a Centrifugal Pump Impeller," by R. D. Bowerman, California Institute of Technology, Hydrodynamics Laboratory Report No. E-19.7, March, 1955. 